\title{
Detailed Studies of Full-Size ATLAS12 Sensors
}

L.B.A. Hommels ${ }^{\mathrm{c}, *}$, P.P. Allport ${ }^{\mathrm{a}}$, M. Baca ${ }^{\mathrm{a}}$, J. Broughton ${ }^{\mathrm{a}}$, A. Chisholm ${ }^{\mathrm{a}}$, K. Nikolopoulos ${ }^{\mathrm{a}}$, S. Pyatt $^{\mathrm{a}}$, J.P. Thomas ${ }^{\mathrm{a}}$, J.A. Wilson ${ }^{\mathrm{a}}$,

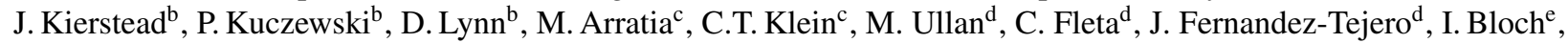

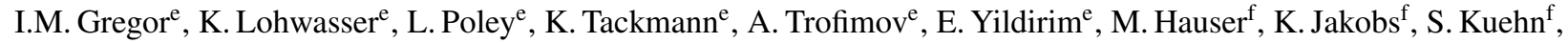

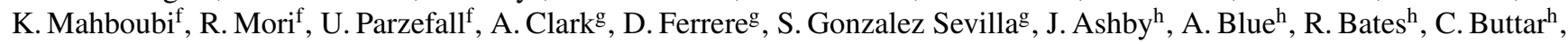

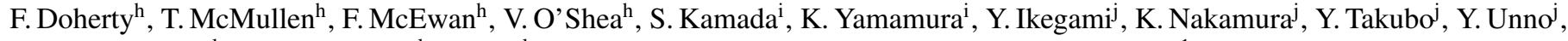
R. Takashima ${ }^{\mathrm{k}}$, A. Chilingarov ${ }^{1}$, H. Fox ${ }^{1}$, A.A. Affolder ${ }^{\mathrm{m}}$, G. Casse ${ }^{\mathrm{m}}$, P. Dervan ${ }^{\mathrm{m}}$, D. Forshaw ${ }^{\mathrm{m}, 1}$, A. Greenall ${ }^{\mathrm{m}}$, S. Wonsak ${ }^{\mathrm{m}}$,

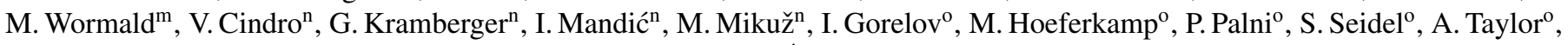
K. Toms ${ }^{\mathrm{o}}$, R. Wang ${ }^{\mathrm{o}}$, N.P. Hessey ${ }^{\mathrm{p}}$, N. Valencic ${ }^{\mathrm{p}}$, K. Hanagaki $^{\mathrm{q}, \mathrm{j}}$, Z. Dolezal ${ }^{\mathrm{r}}$, P. Kodys ${ }^{\mathrm{r}}$, J.Bohm $^{\mathrm{s}}$, J.Stastny ${ }^{\mathrm{s}}$, M.Mikestikova ${ }^{\mathrm{s}}$, A. Bevan ${ }^{\mathrm{t}}$, G. Beck ${ }^{\mathrm{t}}$, C. Milke ${ }^{\mathrm{u}}$, M. Domingo ${ }^{\mathrm{u}}$, V. Fadeyev ${ }^{\mathrm{u}}$, Z. Galloway ${ }^{\mathrm{u}}$, D. Hibbard-Lubow ${ }^{\mathrm{u}}$, Z. Liang ${ }^{\mathrm{u}}$, H. F.-W. Sadrozinski ${ }^{\mathrm{u}}$, A. Seiden ${ }^{\mathrm{u}}$, K. To ${ }^{\mathrm{u}}$, R. French ${ }^{\mathrm{v}}$, P. Hodgson ${ }^{\mathrm{v}}$, H. Marin-Reyes ${ }^{\mathrm{v}}$, K. Parker ${ }^{\mathrm{v}}$, O. Jinnouchi ${ }^{\mathrm{w}}$, K. Hara ${ }^{\mathrm{x}, \mathrm{y}}$, K. Sato ${ }^{\mathrm{x}}$, K. Sato ${ }^{\mathrm{x}, \mathrm{y}}$, M. Hagihara ${ }^{\mathrm{x}}$, S. Iwabuchi ${ }^{\mathrm{x}}$, J. Bernabeu ${ }^{\mathrm{z}}$, J.V. Civera ${ }^{\mathrm{z}}$, C. Garcia ${ }^{\mathrm{z}}$, C. Lacasta ${ }^{\mathrm{z}}$, S. Marti i Garcia ${ }^{\mathrm{z}}$, D. Rodriguez ${ }^{\mathrm{z}}$, D. Santoyo ${ }^{\mathrm{z}}$, C. Solaz ${ }^{\mathrm{z}}$, U. Soldevilaz

\author{
${ }^{a}$ School of Physics and Astronomy, University of Birmingham, Birmingham B15 2TT, United Kingdom \\ ${ }^{b}$ Brookhaven National Laboratory, Physics Department and Instrumentation Division, Upton, NY 11973-5000, USA \\ ${ }^{c}$ Cavendish Laboratory, University of Cambridge, JJ Thomson Avenue, Cambridge CB3 OHE, United Kingdom \\ ${ }^{d}$ Centro Nacional de Microelectronica (IMB-CNM, CSIC), Campus UAB-Bellaterra, 08193 Barcelona, Spain \\ ${ }^{e}$ DESY, Notkestrasse 85, 22607 Hambrug, Germany \\ ${ }^{f}$ Physikalisches Institut, Universität Freiburg, Hermann-Herder-Str. 3, D-79104 Freiburg, Germany \\ ${ }^{g}$ DPNC, University of Geneva, 24, Quai Ernest-Ansermet, CH-1211 Genve 4, Switzerland \\ ${ }^{h}$ SUPA- School of Physics and Astronomy, University of Glasgow, Glasgow G12 8QQ, United Kingdom \\ ${ }^{i}$ Solid State Div., Hamamatsu Photonics K.K., 1126-1, Ichino-cho, Higashi-ku, Hamamatsu-shi, Shizuoka 435-8558, Japan \\ ${ }^{j}$ Institute of Particle and Nuclear Study, KEK, Oho 1-1, Tsukuba, Ibaraki 305-0801, Japan \\ ${ }^{k}$ Department of Science Education, Kyoto University of Education, Kyoto 612-8522, Japan \\ ${ }^{l}$ Physics Department, Lancaster University, Lancaster LA1 4YB, United Kingdom \\ ${ }^{m}$ Oliver Lodge Laboratory, Department of Physics, University of Liverpool, Oxford St., Liverpool L69 7ZE, United Kingdom \\ ${ }^{n}$ Jožef Stefan Institute and Department of Physics, University of Ljubljana, Ljubljana, Slovenia \\ ${ }^{o}$ Department of Physics and Astronomy, University of New Mexico, MSC07 4220, 1919 Lomas Blvd. NE, Albuquerque, NM 87131, USA \\ ${ }^{p}$ Nikhef, Science Park 105, 1098 XG Amsterdam, Netherlands \\ ${ }^{q}$ Department of Physics, Osaka University, Machikaneyama-cho 1-1, Toyonaka-shi, Osaka 560-0043, Japan \\ ${ }^{r}$ Charles University in Prague, Faculty of Mathematics and Physics, V Holesovickach 2, Prague 8, Czech Republic \\ ${ }^{s}$ Academy of Sciences of the Czech Republic, Institute of Physics, Na Slovance 2, 18221 Prague 8, Czech Republic \\ ${ }^{t}$ School of Physics and Astronomy, Queen Mary University of London, London E1 4NS, United Kingdom \\ ${ }^{u}$ Santa Cruz Institute for Particle Physics (SCIPP), University of California, Santa Cruz, CA 95064, USA \\ ${ }^{v}$ Department of Physics and Astronomy, The University of Sheffield, Hicks Building, Hounsfield Road, S3 7RH Sheffield, United Kingdom \\ ${ }^{w}$ Institute of Science and Engineering, Tokyo Institute of Technology, Ookayama 2-12-1, Meguro-ku, Tokyo 152-8551, Japan \\ ${ }^{x}$ Institute of Pure and Applied Sciences, University of Tsukuba, Tsukuba, Ibaraki 305-8751, Japan \\ ${ }^{y}$ Center for Integrated Research in Fundamental Science and Engineering, University of Tsukuba, Tsukuba, Ibaraki 305-8571 Japan \\ ${ }^{z}$ IFIC/CSIC-UVEG, Ed. Inst. Investigacion, PO Box 22085, 46071 Valencia, Spain
}

\begin{abstract}
The "ATLAS ITk Strip Sensor Collaboration" R\&D group has developed a second iteration of single-sided $\mathrm{n}^{+}$-in-p type microstrip sensors for use in the tracker upgrade of the ATLAS experiment at the High-Luminosity (HL) LHC. The full size sensors measure approximately $97 \times 97 \mathrm{~mm}^{2}$ and are designed for tolerance against the $1.1 \times 10^{15} \mathrm{n}_{\mathrm{eq}} / \mathrm{cm}^{2}$ fluence expected at the HLLHC. Each sensor has 4 columns of 1280 individual $23.9 \mathrm{~mm}$ long channels, arranged at $74.5 \mu \mathrm{m}$ pitch. Four batches comprising of 120 sensors produced by Hamamatsu Photonics were evaluated for their mechanical, and electrical bulk and strip characteristics. Optical microscopy measurements were performed to obtain the sensor surface profile. Leakage current and bulk capacitance properties were measured for each individual sensor. For sample strips across the sensor batches, the inter-strip capacitance and resistance as well as properties of the punch-through protection structure were measured. A multi-channel probecard was used to measure leakage current, coupling capacitance and bias resistance for each individual channel of 100 sensors in three batches. The compiled results for 120 unirradiated sensors are presented in this paper, including summary results for almost 500 '000 strips probed. Results on the reverse bias voltage dependence of various parameters and frequency dependence of tested capacitances are included for validation of the experimental methods used. Comparing results with specified values, almost all sensors fall well within specification.
\end{abstract}

Keywords: HL-LHC, ATLAS ITk, Micro-strip Sensor, Leakage Current, Depletion, Strip Test 


\section{Introduction}

The foreseen upgrade of the Large Hadron Collider (LHC) to the High-Luminosity LHC (HL-LHC) is scheduled to deliver collisions in 2022 [1]. To achieve a total cross section of $3000 \mathrm{fb}^{-1}$, the instantaneous luminosity of the HL-LHC is expected to reach $5 \times 10^{34} \mathrm{~cm}^{-2} \mathrm{~s}^{-1}$ at a centre of mass energy of $14 \mathrm{TeV}$. The increase in particle fluence necessitates an upgrade of the ATLAS inner detector: an all-silicon new inner tracker (ITk) [2] is proposed to replace the current SemiConductor Tracker (SCT) and Transition Radiation Tracker (TRT). The ITk layout as presented in the Letter of Intent [3] assumes silicon microstrip detectors to be used for 7 endcap disks, and 5 barrel layers. From simulations verified by experiments, the highest particle fluence in the barrel short strip layer is expected to be $5.3 \times 10^{14} \mathrm{n}_{\mathrm{eq}} / \mathrm{cm}^{2}$ [4]. Including a safety factor of 2 , candidate ITk sensors will have to be radiation hard up to levels of $1.1 \times 10^{15} \mathrm{n}_{\mathrm{eq}} / \mathrm{cm}^{2}$. The goal of the ITk Strip Sensor collaboration is to develop a silicon microstrip sensor that is suitable for use in the new ITk. Results of detailed studies of properties of full-size sensor prototypes are presented in this paper, whereas studies of radiation damage of $1 \times 1 \mathrm{~cm}^{2}$ miniature sensors are reported in [5], [6].

\section{ATLAS12 Large Area Sensors}

The ATLAS12 sensors are the second iteration of sensors designed for the Upgrade ITk, superseding the ATLAS07 types [7]. To cope with the effects of radiation damage during the sensor lifetime, operation in partial depleted mode is foreseen towards the end of the detector lifetime. The sensor will need specially designed structures between the strips to guarantee strip isolation during its lifetime and mitigate radiation-induced surface damage whilst retaining a low inter-strip capacitance. A single-sided $\mathrm{n}^{+}$-in-p sensor offers a trade-off between the above requirements and cost [8]. 120 full-size prototype sensors were manufactured by Hamamatsu Photonics [9] on a 6" wafer process using p-type float zone silicon. Sensors were delivered in 4 batches: VPX12318, VPX12518, and VPX12519, of 33, 32 and 35 sensors respectively were shipped to the UK, and VPX14757 containing 20 sensors was delivered to the US.

The largest square sensor that can be cut from the wafer measures $97.5 \times 97.5 \mathrm{~mm}^{2}$. This geometry is denoted "Outer Cut". Sensors with $462 \mu \mathrm{m}$ reduced edge metal all around, denoted "Inner Cut", were made available as well to allow for smaller inactive regions in the final detector layout. The sensor strip implants are arranged in 4 columns of 1282 implants each, with the top and bottom implant of each column serving as field shaping strips. The resulting strip pitch is $74.5 \mu \mathrm{m}$, the strip length is $23.9 \mathrm{~mm}$. All strips are connected to the bias rail by poly-silicon resistors implanted in the sensor. For biasing the central two columns, a bias rail runs through the center of the sensor. Top metal layer strips are AC-coupled to the strip

\footnotetext{
${ }^{*}$ Corresponding author. e-mail: lbah2@cam.ac.uk

${ }^{1}$ Now at Syracuse University.
}

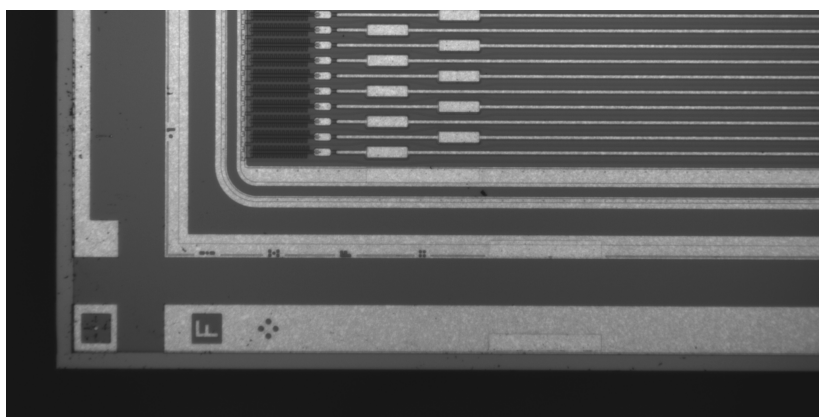

Fig. 1: Picture of the corner of an Outer Cut ATLAS12 sensor, illustrating the layout of the bias rail, guard ring, strip top metal and bias resistors. The dicing streets intended for the Inner Cut sensors are clearly visible.

implants, which have p-stop traces running in between along the full length for strip isolation. The top metal layer is passivated, with openings for probing and wirebonding. Figure 1 contains a photograph showing the sensor details.

\section{ATLAS12A mechanical properties}

The sensor mechanical specifications state the following:

- nominal thickness: $310 \pm 20 \mu \mathrm{m}$,

- thickness variation: $\pm 10 \mu \mathrm{m}$ across the sensor. This means some thickness variation is allowed between sensors, but not across a sensor.

- sensor flatness when unstressed: $<200 \mu \mathrm{m}$,

- Outer Cut dimensions: $97540 \pm 25 \mu \mathrm{m}$ square,

- Inner Cut dimensions: $95692 \pm 25 \mu \mathrm{m}$ square, and

- no cracks or chips at the dicing line to extend further inwards than $50 \mu \mathrm{m}$.

For visual inspection and evaluation of the above properties, a non-contact optical Coordinate Measurement Machine (CMM) was used, with a precision better than $2 \mu \mathrm{m}$ in $x, y$, and $4 \mu \mathrm{m}$ in $z$. A coordinate system is defined, with the origin $(0,0,0)$ at the centre of the sensor, with the axes in righthanded orientation with the $z$ axis pointing upwards, and the scratch pad located in the bottom right. For measuring outer
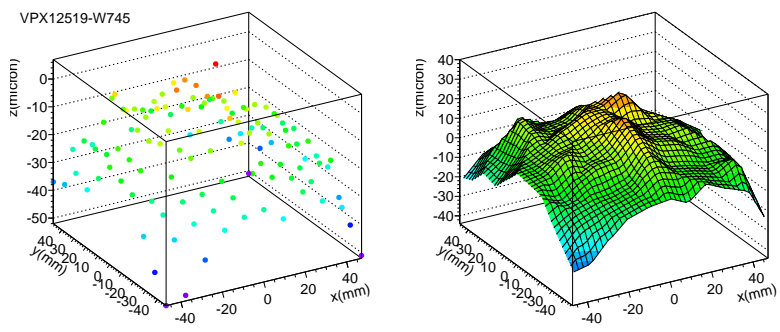

Fig. 2: Measured space points (left), and net bow interpolated plot (right) for sensor VPX12519-W745. 


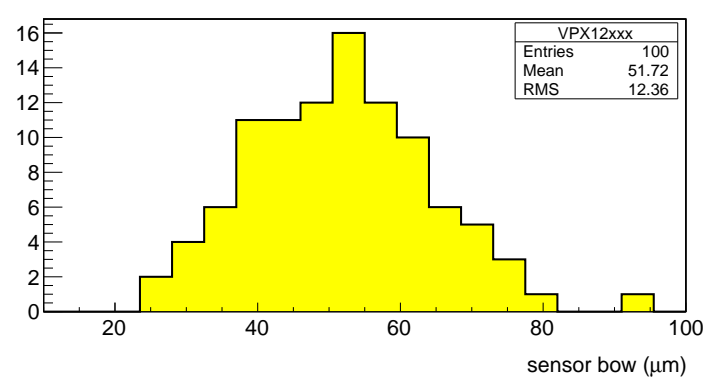

Fig. 3: Histogram of the maximum bow of the sensors from batches VPX12318, VPX12518 and VPX12519.

and inner cut sensors within a reasonable timespan, measurements were taken on a $11 \times 11$ grid with $9.440 \mathrm{~mm}$ spacing. To compensate for tilt of the freely suspended sensor, a flat plane is fitted to, and subtracted from the space points measured. The measurement points, and an interpolated net bow result for a typical sensor are plotted in Fig. 2. The difference between the highest and lowest points from the net bow is taken as the maximum bow. A histogram of the maximum bow of sensors from batches VPX12318, VPX12518 and VPX12519 is plotted in Fig. 3, the resulting average is $51.7 \mu \mathrm{m}$, with an RMS deviation of $12.4 \mu \mathrm{m}$, which is well within specification.

\section{Bias Voltage and Frequency dependence of parameters}

\subsection{Leakage Current}

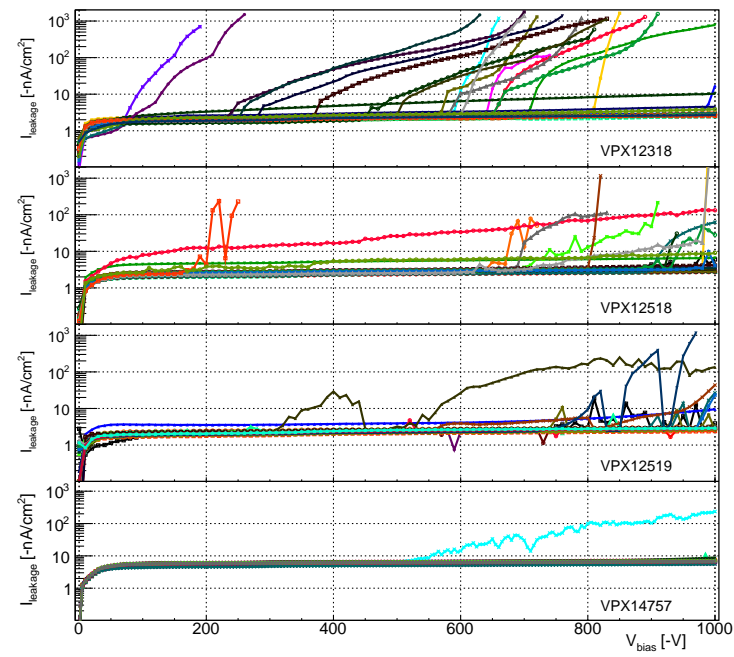

Fig. 4: Leakage currents versus $\mathrm{V}_{\text {bias }}$ for ATLAS12 sensors at $21^{\circ} \mathrm{C}$, grouped per batch.

Development of the leakage current against bias voltage $\left(\mathrm{V}_{\text {bias }}\right)$ for all 120 sensors is plotted in Fig. 4. The data was taken at $\mathrm{T}=21^{\circ} \mathrm{C}$, and at a relative humidity $(\mathrm{RH})$ of $40 \pm 10 \%$. Since the ATLAS12 sensors are very sensitive to the ambient humidity, the initial batch VPX12318 was tested in a dry $\mathrm{N}_{2}$ environment. As can be observed from the graphs on VPX12318 however, a considerable number of sensors exhibit early, soft

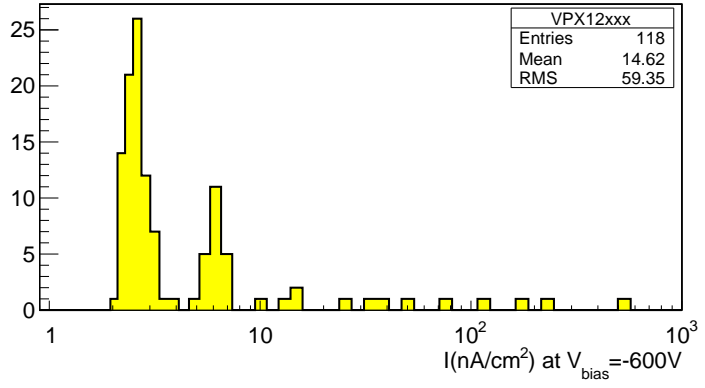

Fig. 5: Histogram of the normalised leakage current at $\mathrm{V}_{\text {bias }}=-600 \mathrm{~V}$, at $\mathrm{T}=21^{\circ} \mathrm{C}$.

breakdown. The two subsequent batches were stored in dry atmosphere, and measured in standard cleanroom conditions at $\mathrm{T}=21^{\circ} \mathrm{C}$, and $40 \pm 10 \% \mathrm{RH}$, and fewer soft breakdowns occur. Measurements were carried out using Keithley 2410 and 6517 Source Measure Units (SMUs), with 10V step increases with a 10 s delay from $0 \mathrm{~V}$ to $-1000 \mathrm{~V}$ unless the compliance limit of $100 \mu \mathrm{A}$ was exceeded. In some cases, the sensors were trained by increasing the bias voltage very slowly, after which a normal IV measurement was recorded.

With a $2 \mu \mathrm{A} / \mathrm{cm}^{2}$ specification for leakage current, and the area inside the bias ring spanning $91.8 \mathrm{~cm}^{2}$, the maximum allowed leakage current at $-600 \mathrm{~V}$ bias and $\mathrm{T}=21^{\circ} \mathrm{C}$, is $184 \mu \mathrm{A}$. Two sensors exhibited early breakdown, and were excluded from all further measurements. A histogram containing the leakage currents at $\mathrm{V}_{\text {bias }}=-600 \mathrm{~V}$ of the remaining 118 sensors is plotted in Fig. 5. It shows that the sensors examined sit well below $1 \mu \mathrm{A} \mathrm{cm} \mathrm{cm}^{2}$, comfortably within specifications.

\subsection{Bulk Capacitance}

The sensor bulk capacitance $\left(\mathrm{C}_{\text {bulk }}\right)$ was measured as a function of bias voltage to determine the sensor depletion, and versus frequency to verify the validity of the measurement. Using a Wayne-Kerr 6440B LCR meter in R-C series network mode, the frequency dependence of $\mathrm{C}_{\text {bulk }}$ for various $\mathrm{V}_{\text {bias }}$ values of sensor VPX12519-W748 is plotted in Fig. 6. As can be observed, the $\mathrm{C}_{\text {bulk }}$ is constant between 0.5 and $6 \mathrm{kHz}$ for a wide range of $\mathrm{V}_{\text {bias }}$. $\mathrm{C}_{\text {bulk }}$ measurements were carried out with frequencies between 1 and $2 \mathrm{kHz}$.

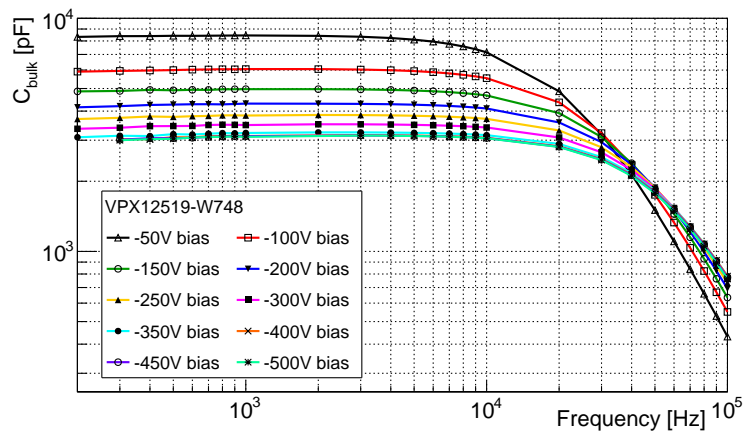

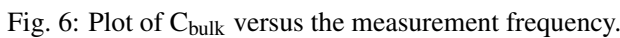




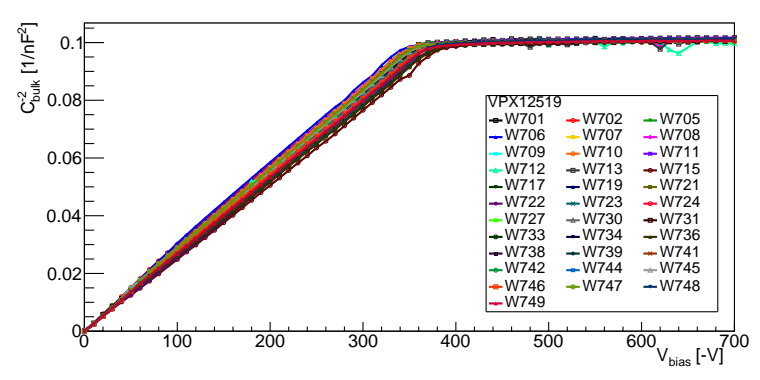

Fig. 7: Plot of $1 / \mathrm{C}_{\text {bulk }}{ }^{2}$ versus $\mathrm{V}_{\text {bias }}$ for the sensors of batch VPX12519

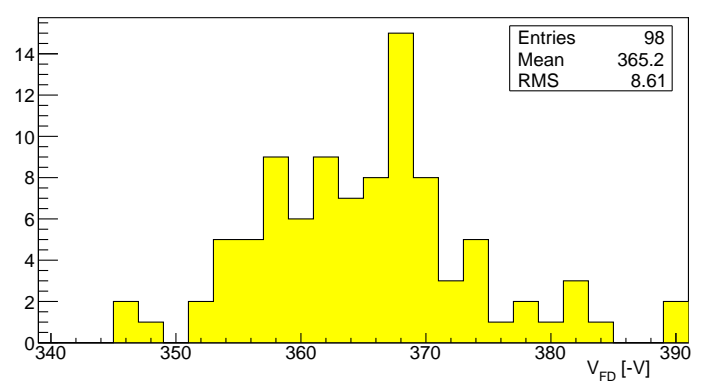

Fig. 8: Histogram of $\mathrm{V}_{\mathrm{FD}}$ determined from $\mathrm{C}_{\text {bulk }}$, see text.

Figure 7 contains plots of $1 / \mathrm{C}_{\text {bulk }}^{2}$ versus $\mathrm{V}_{\text {bias }}$ for 33 sensors of batch VPX12519. As can be seen from the plot, behaviour is very consistent within the batch. The full depletion voltage $\left(\mathrm{V}_{\mathrm{FD}}\right)$ is determined by taking the intersection point of straight line fits to the sloped, and flat region. Histogrammed $\mathrm{V}_{\mathrm{FD}}$ for 98 sensors of batches VPX12318, VPX12518 and VPX12519 are plotted in Fig. 8: the distribution is narrow, with an average of $\mathrm{V}_{\mathrm{FD}}=365 \mathrm{~V}$, and a RMS of $8.6 \mathrm{~V}$.

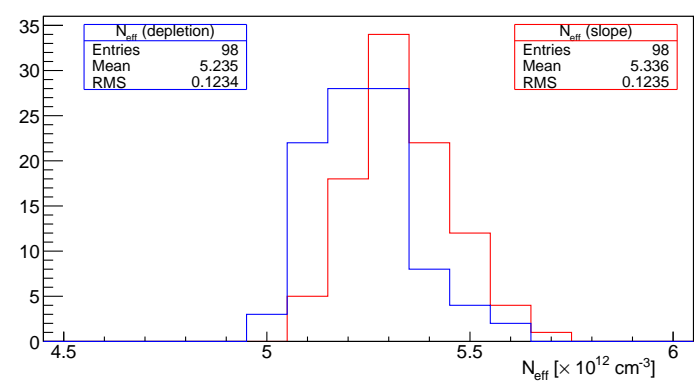

Fig. 9: Histogrammed doping concentrations $\mathrm{N}_{\mathrm{eff}}$, extracted from the data plotted in Fig. 7. The blue line histogram is extracted from the flat region, the red line histogram is from the sloped region.

Assuming a parallel plate capacitor model for the sensor, with the area defined as the inside of the bias ring, the active thickness is estimated at $301 \mu \mathrm{m}$. With the area and thickness known, the doping concentration can be calculated both from the sloped region as well as the saturated region of the $C_{b u l k}$ data. For both methods, the calculated doping concentrations are plotted with blue and red lines respectively in Fig. 9. The resulting values of $5.3 \pm 0.12 \times 10^{12} \mathrm{~cm}^{-3}$ are consistent with a wafer bulk resistivity of $2.5 \mathrm{k} \Omega \mathrm{cm}$, which is, although the $\mathrm{V}_{\mathrm{FD}}$ is higher than the desired value of $<300 \mathrm{~V}$, in accordance with specification.

\subsection{Strip Coupling Capacitance}
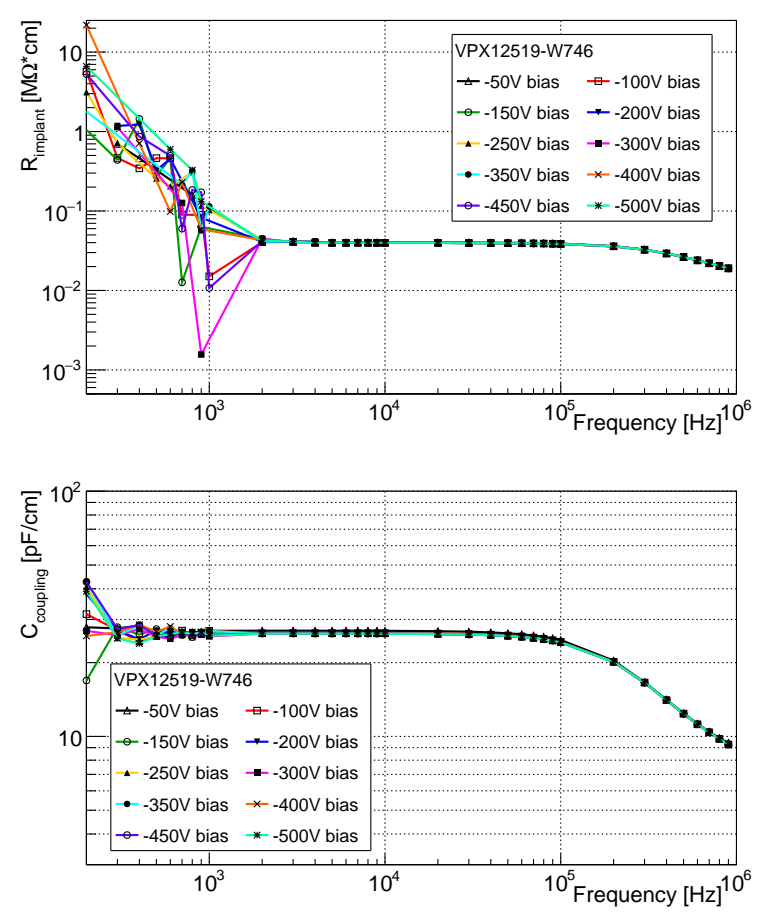

Fig. 10: Frequency dependence of $\mathrm{R}_{\text {implant }}$ (top plot), and $\mathrm{C}_{\text {coupling }}$ (bottom plot), for various $\mathrm{V}_{\text {bias }}$ values.

The capacitance and resistance of the R-C network formed by the strip implant resistance $\left(\mathrm{R}_{\text {implant }}\right)$ and the capacitance between the implant and the top metal readout strip $\left(\mathrm{C}_{\text {coupling }}\right)$ are plotted as a function of measurement frequency for various bias voltages in Fig. 10. It has been measured directly between the strip implant and top metal using an LCR in R-C series network mode. As can be seen from the plots, the behaviour of the capacitance, and implant resistance both show little variation across a $2-100 \mathrm{kHz}$ range. At higher frequencies, the resistance and capacitance drop, as expected from the implant resistance and the transmission line formed between the strip implant and top metal. The nominal value of $\mathrm{C}_{\text {coupling }}$ is $25.7 \mathrm{pF} / \mathrm{cm}$, which is well within the specification of $>20 \mathrm{pF} / \mathrm{cm}$. More results are presented and discussed in Section 5.

\subsection{Bias Resistance}

The bias resistance ( $R_{\text {bias }}$ ) for a small sample of channels was measured directly, as part of the DC measurement of the Punch-Through Protection circuit behaviour, see Section 4.7. The current between the implant and the bias rail was monitored while the voltage was increased from $0 \mathrm{~V}$ to $10 \mathrm{~V}$ in $0.25 \mathrm{~V}$ steps. The results lie within $1.5 \pm 0.03 \mathrm{M} \Omega$, which is well within the specification of $R_{\text {bias }}=1.5 \pm 0.5 \mathrm{M} \Omega$. More results on many individual strips probed are presented and discussed in Section 5 . 


\subsection{Inter-Strip Capacitance}

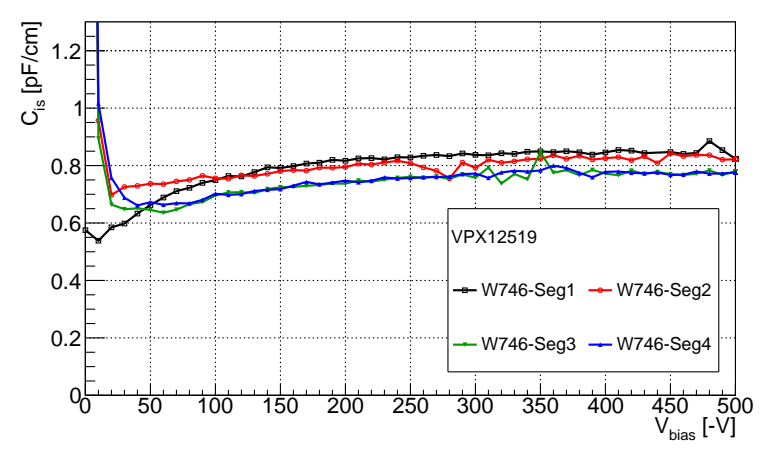

Fig. 11: Inter-strip capacitance $C_{\text {is }}$ result for a few sample strips versus $V_{\text {bias }}$.

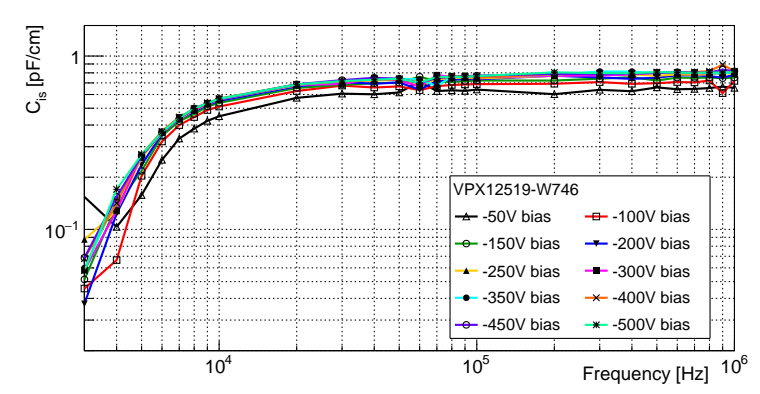

Fig. 12: Behaviour of the inter-strip capacitance $C_{\text {is }}$ versus measurement frequency for various $\mathrm{V}_{\text {bias }}$ values.

The inter-strip capacitance $\left(\mathrm{C}_{\text {is }}\right)$ dominates the input capacitance contributions to the front-end electronics, and determines the noise levels of the detector. Its value is one of the main input parameters to the design process of new revisions of the front-end preamplifier, and should be kept as low as possible. The specification states it should be $<0.8 \mathrm{pF} / \mathrm{cm}$, measured at $100 \mathrm{kHz}$ with both neighbouring strips grounded. For ATLAS12 sensors with $23.9 \mathrm{~mm}$ strip length, $\mathrm{C}_{\mathrm{is}}<1.91 \mathrm{pF}$. The development of $\mathrm{C}_{\text {is }}$ versus $\mathrm{V}_{\text {bias }}$ for 4 sample channels is plotted in Fig. 11. The $C_{\text {is }}$ value is largely determined by the strip metal geometry, and sensor surface layout. Its frequency dependence is plotted in Fig. 12, results suggesting the measurement frequency should lie in the $50 \mathrm{kHz}$ to $1 \mathrm{MHz}$ range. The average value measured is $0.77 \mathrm{pF} / \mathrm{cm}$, confirming findings from other institutes.

\subsection{Inter-Strip Resistance}

The inter-strip resistance $\left(\mathrm{R}_{\mathrm{is}}\right)$ was measured by applying a master voltage $\left(\mathrm{V}_{\mathrm{M}}\right)$ to a strip implant, and measuring the induced current $\left(i_{c}\right)$ on the neighbouring strip by sampling the voltage developed across its bias resistor $\mathrm{V}_{\mathrm{S}}$. $\mathrm{V}_{\mathrm{M}}$ is applied in $1 \mathrm{~V}$ steps in the interval $[-5 \mathrm{~V},+5 \mathrm{~V}]$. Even though instruments with high input impedance and excellent isolation were used (Keithley 6517, Keithley 2000), it is likely that above 10-20 G $\Omega$ the accuracy is limited due to setup systematics. Nonetheless, from the plot of $\mathrm{V}_{\mathrm{S}}$ against $\mathrm{V}_{\mathrm{M}}$ in the top plot in Fig. 13, it
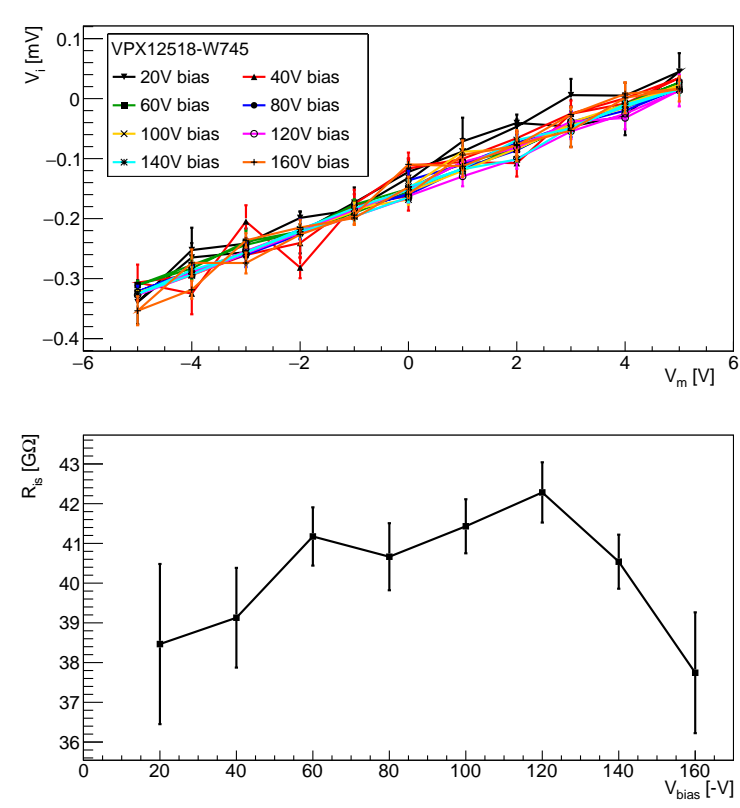

Fig. 13: Top plot: measured voltage induced by $i_{c}$ against the applied voltage $V_{M}$ for various values of $V_{\text {bias }}$ (see text). Bottom plot: extracted $R_{\text {is }}$ against bias voltage.

shows that there is a clear linear dependence of the two properties. The bottom plot contains summary results on extracted $\mathrm{R}_{\text {is }}$ for sample strips for a range of $\mathrm{V}_{\text {bias }}$.

\subsection{Punch-Through Protection}

To protect the strip implants and front-end electronics from excessive charge liberated in the bulk, for example as the result of a beam splash, a Punch-Through Protection (PTP) structure is incorporated in the sensor. It embodies an elongation of the strip implant under the bias resistor, leaving a $8 \mu \mathrm{m}$ gap to the bias rail. Soft breakdown will occur across this gap as soon as a threshold potential is exceeded. The PTP structure is of the "fully gated" design, where the bias rail implant extends over the gap, covering the PTP gap entirely, see [5].

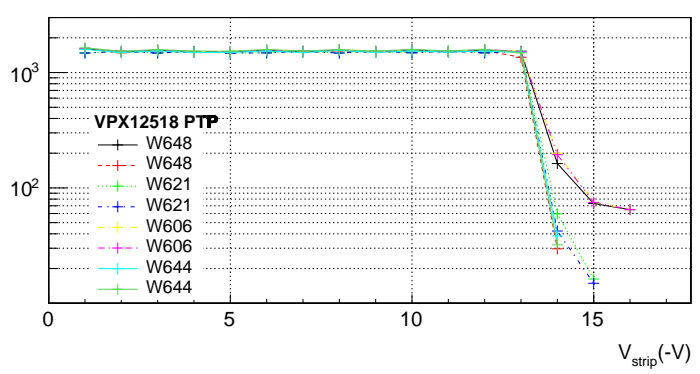

Fig. 14: Plot showing the apparent resistance $\Delta \mathrm{V} / \Delta \mathrm{I}$ against the voltage applied to the strip implant $\mathrm{V}_{\text {strip. }}$.

A static measurement to determine the threshold voltage consists of monitoring the current whilst increasing the voltage imposed on the strip implant. To limit the power dissipation, voltage and current are limited to $50 \mathrm{~V}, 50 \mu \mathrm{A}$ respectively. 
Typical results for sample channels of the differentiated $\mathrm{dV} / \mathrm{dI}$ plot, showing the resistance against applied voltage are plotted in Fig. 14. A threshold at $13 \mathrm{~V}$ is clearly visible, with the apparent resistance dropping sharply from the $1.5 \mathrm{M} \Omega$ bias resistance to tens of $\mathrm{k} \Omega$. As expected, the sharper drops are observed when the voltage is applied on the implant end nearer to the PTP structure.

\section{Strip Scan Results}

\subsection{Measurement Techniques}

To evaluate properties of individual strips, study the uniformity of electrical characteristics over the entire sensor surface, and compare against other sensors, full scans of individual sensor channels were made. A relatively simple Strip Test protocol, see the following Section 5.2, was used to check for strip shorts and pinholes, and measure the $\mathrm{C}_{\text {coupling }}$ and $\mathrm{R}_{\text {bias }}$ for each individual strip. A single probe needle was used for probing the initial 31 sensors in batch VPX12318, after that a custom 32 channel probecard and associated multiplexing equipment were used, reducing the sensor measurement time from 14 to under 3 hours

During the test, the sensor was held in place by a low-force spring rather than a vacuum jig to minimise stress and strain on the sensor that could lead to early breakdown or influence strip test results, with the bias provided by wirebonded contacts to the edge metal and bias rail. Additional needles on the probecard provide a edge-sense feature, breaking a contact as soon as the needles touch the sensor surface. After detecting touchdown, the $z$-height is recorded and a pre-defined probestation chuck overdrive is applied to ensure a good contact between the probecard needles and the sensor. This procedure was repeated for every touchdown, providing uniform and consistent contact quality across the entire sensor. In contrast, for the single-needle measurement such functionality does not exist, resulting in variable contact quality due to the non-planarity of the prober chuck, jig or sensor.

\subsection{Strip Test Description}

A "Full Strip Test" refers to the following sequence for testing a single strip on a sensor that is biased at $-150 \mathrm{~V}$ to achieve full strip isolation:

- Connect to the strip metal using a probe needle.

- Connect an SMU set to $10 \mathrm{~V}$ to the strip metal through a $1 \mathrm{M} \Omega$ current-limiting series resistance, and measure the current.

- If the current exceeds $1 \mu \mathrm{A}$, the strip is marked as having a short, and the measurement proceeds to the next strip.

- The SMU voltage is increased to $100 \mathrm{~V}$, and the current is recorded after a short time delay.

- The strip is connected to GND, and subsequently to the LCR meter.
- The LCR meter measures the R-C series network formed by $\mathrm{R}_{\text {bias }}$ and $\mathrm{C}_{\text {coupling }}$, and results are recorded.

- The strip metal is connected to GND and the measurement proceeds to the next strip.

\subsection{Strip Test Results Summary}

Histograms of accumulated data of strip metal current (top

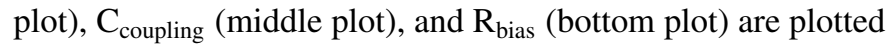
in Fig. 15. Data from almost 500000 channels from 97 sensors from batches VPX12318, VPX12518, and VPX12519 is included. One sensor exhibited high leakage current and was
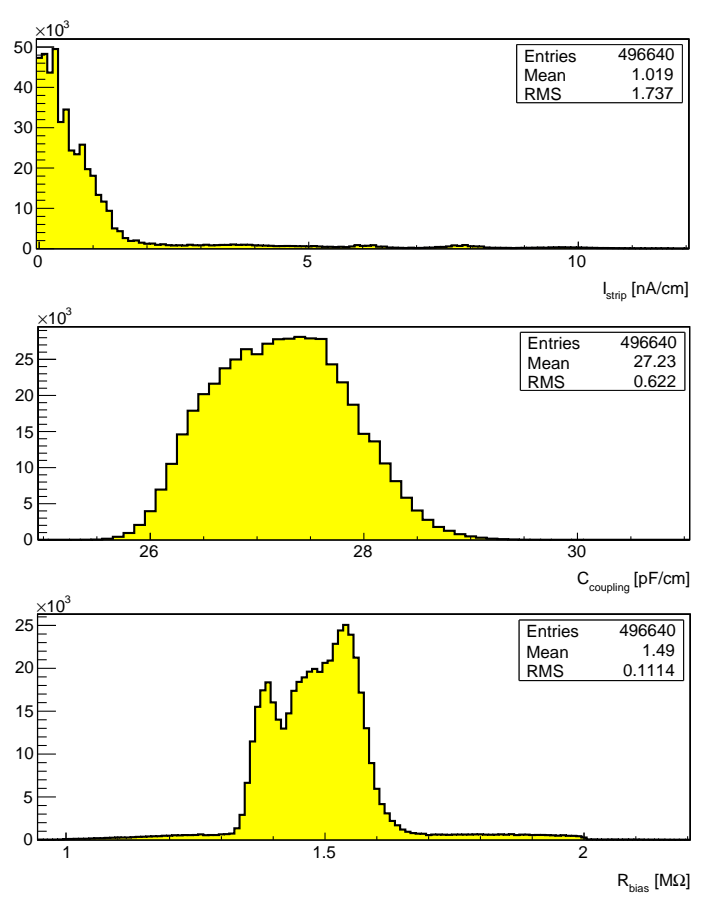

Fig. 15: Accumulated strip test results for 97 sensors from batches VPX12318, VPX12518, and VPX12519.

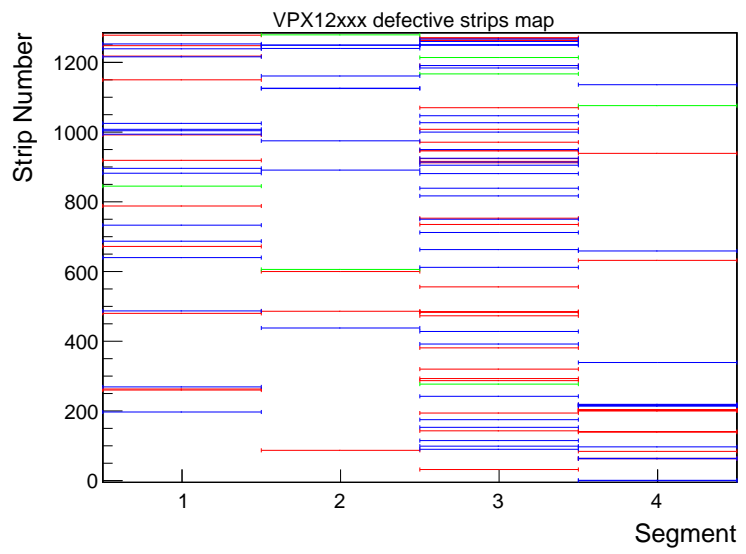

Fig. 16: Map of 132 faulty strips accumulated from 97 measured sensors. Strips failing from $\mathrm{R}_{\text {bias }}, \mathrm{C}_{\text {coupling, }}$, and strip current are plotted in red, green and blue, respectively. 


\begin{tabular}{l|l|l} 
Sensor Property & Specification & Measured values \\
\hline Maximum Bow & $<200 \mu \mathrm{m}$ & $51.7 \pm 12.4 \mu \mathrm{m}$ \\
Leakage current at $\mathrm{V}_{\text {bias }}=600 \mathrm{~V}$ & $<2 \mu \mathrm{A} / \mathrm{cm}^{2}$ & $<1 \mu \mathrm{A} / \mathrm{cm}^{2}$ for 118 of 120 sensors \\
Depletion Voltage & $<300 \mathrm{~V}$, wafer resistivity allowing & $365 \pm 8.6 \mathrm{~V}$ \\
Bias Resistor Value & $1.5 \pm 0.5 \mathrm{M} \Omega$ & $1.49 \pm 0.111 \mathrm{M} \Omega$ \\
Coupling Capacitance & $>20 \mathrm{pF} / \mathrm{cm}$ at $\mathrm{V}_{\text {bias }}=300 \mathrm{~V}$ & $27.2 \pm 0.62 \mathrm{pF} / \mathrm{cm}$ \\
Inter-Strip Capacitance & $<0.8 \mathrm{pF} / \mathrm{cm}$ & $0.77 \mathrm{pF} / \mathrm{cm}$ \\
Inter-Strip Resistance & $>15 \pm 5 \mathrm{M} \Omega$ & $>30 \mathrm{G} \Omega$ \\
Percentage Good Strips & $>98 \%$ per segment & $>99.97$
\end{tabular}

Table 1: Comparison of ATLAS12 specification and summarized measurement values for various sensor properties.

excluded from the measurement. Ignoring defective channels, the measured values fall well within specification, see Table 1.

A geometrical map of accumulated defective channels is displayed in Fig. 16. There seems to be no obvious correlation with sensor geography, although segment 3 seems to have significantly more defects than the other segments. Of the 132 recorded faulty channels across 97 sensors probed, 79 have a short or pinhole, 10 fail the $\mathrm{C}_{\text {coupling }}$ requirement, and of 43 strips the $\mathrm{R}_{\text {bias }}$ is out of range. The total fraction of good strips is $99.97 \%$, comfortably exceeding the $98 \%$ requirement.

\section{Summary and Conclusion}

Detailed studies on ATLAS12 sensors produced by Hamamatsu Photonics has been presented in this paper. Results from the evaluation are summarized in Table 1, and compared to the specifications set out in the ATLAS12 Technical Specification Document. 118 of 120 sensors tested satisfy the specifications for non-irradiated sensors for maximum bow, leakage current. Only $0.03 \%$ of probed strips were measured as defective; all other strips satisfied the requirements for $\mathrm{C}_{\text {coupling }}$ and $\mathrm{R}_{\text {bias }}$. The values of the Inter-Strip Capacitance and -Resistance measured for sample channels are well within specification. The conclusion of examination of 120 sensors is that they are of high quality, have good consistency between sensors in three different batches, and excellent uniformity across channels on a sensor. They satisfy the requirements, and are considered suitable for use in the barrel of the ATLAS Upgrade ITk.

\section{Acknowledgements}

The Irradiations were performed: with protons at the University of Birmingham MC40 cyclotron, supported by the H2020 project AIDA-2020, GA number 654168, and the UK's Science and Technology Facilities Council, at Cyclotron and Radioisotope Center (CYRIC), Tohoku University, with Y. Sakemi, M. Ito, and T. Wakui, at the Karlsruhe Institute of Technology (KIT) by A. Dierlamm, supported by the Initiative and Networking Fund of the Helmholtz Association, contract HA-101 (Physics at the Terascale) and the European Commission under the FP7 Research Infrastructures project AIDA, Grant agreement no.262025, and at the LANSCE facility, Los Alamos National Laboratory; with neutrons at JSI TRIGA reactor in Ljubljana supported by the H2020 project AIDA2020, GA no. 654168; with pions at Paul Scherrer Institut (PSI); and with $\gamma$ 's at Brookhaven National Laboratory (BNL). The research was supported and financed in part by the Ministry of Education, Youth and Sports of the Czech Republic (Grant No. LG13009), the German Federal Ministry of Education and Research, and the Helmholtz Association, the European Social Fund and by the Ministry of Science, Research and Arts, Baden-Wuerttemberg, Germany, the Japan Society for Promoting Science KAKENHI-A Grant number 20244038 and KAKENHI-C Grant number 20540291, the Ministry of Education, Culture, Sports, Science and TechnologyJapan, KAKENHI for Research on Priority Area Grant number 20025007 and for Scientific Research on Innovative Areas Grant number 23104002, the Slovenian Research Agency, the Spanish Ministry of Economy and Competitiveness through the Particle Physics National Program (ref. FPA2012-39055C02-01 and FPA2012-39055-C02-02) and co-financed with FEDER funds, the financial support of the State Secretariat for Education, Research, and Innovation, the Swiss National Science Foundation and the Canton of Geneva, Switzerland, the UK Science and Technology Facilities Council (Grant ST/M006409/1), CONICYT Becas Chile 72140349 and Cambridge Trust, and the United States Department of Energy, grant DE-FG02 13ER41983.

\section{References}

[1] S. McMahon. Lhc upgrade program of accelerators and experiments. This conference, 2015.

[2] I.-M. Gregor. Strips and tracker infrastructure. This conference, 2015.

[3] ATLAS Collaboration. Letter of Intent for the Phase-II Upgrade of the ATLAS Experiment. LHCC-I-023, CERN-LHCC-2012-022, 2012.

[4] I. Dawson P.S. Miyagawa. Radiation background studies for the phase ii inner tracker upgrade. ATL-UPGRADE-PUB-2013-012, 2013.

[5] M. Mikestikova. Study of surface properties of atlas12 strip sensors and their radiation resistance. This conference, 2015.

[6] K. Hara. Charge collection and field profile studies in heavily irradiated silicon strip sensors for the atlas inner tracker upgrade. This conference, 2015.

[7] J. Bohm et al. Evaluation of the bulk and strip characteristics of large area n-in-p silicon sensors intended for a very high radiation environment. $\mathrm{Nucl}$. Instrum. Meth., A636:S104-S110, 2011.

[8] Y. Unno et al. Development of $\mathrm{n}^{+}$-in-p large-area silicon microstrip sensors for very high radiation environments - ATLAS12 design and initial results. Nucl. Instrum. Meth., A765:80-90, 2014.

[9] Hamamatsu Photonics. www.hamamatsu.com. 2015. 